
\title{
25 Research Soure \\ Debulking mastectomy with electrochemotherapy: a case report of no surgery approach to recurrent breast cancer.
}

Emanuela Esposito ( $\nabla$ emanuelaexpo@hotmail.it )

Istituto Nazionale Tumori IRCCS Fondazione Pascale https://orcid.org/0000-0002-6394-8419

Claudio Siani

Istituto Nazionale Tumori IRCCS Fondazione Pascale

Ugo Pace

Istituto Nazionale Tumori IRCCS Fondazione Pascale

Raffaele Costanzo

Istituto Nazionale Tumori IRCCS Fondazione Pascale

Raimondo di Giacomo

Istituto Nazionale Tumori IRCCS Fondazione Pascale

\section{Case report}

Keywords: electrochemotherapy; breast cancer recurrence; case report; mastectomy; multidrug resistance

Posted Date: July 8th, 2020

DOl: https://doi.org/10.21203/rs.3.rs-40425/v1

License: (9) This work is licensed under a Creative Commons Attribution 4.0 International License. Read Full License 


\section{Abstract}

Background Electrochemotherapy has been shown to be safe, effective and non-invasive loco-regional treatment for chest wall breast cancer recurrence. Electrochemotherapy is the gold standard treatment of patients with cutaneous metastases from breast cancer who due to the extent of those lesions are not eligible for resection or radiotherapy has already been used, and systemic therapy is ineffective or contraindicated.

Case presentation Here we present the case of a women affected by multiple metastatic cancers with multi-drug resistance who refused mastectomy after being diagnosed with recurrent ulcerated right breast cancer. We first describe an extended indication to electrochemotherapy to treat breast cancer recurrence after breast conserving surgery. Electroporation-induced necrosis through electrochemotherapy replaced surgery and was delivered in 30 minutes at $5000 \mathrm{~Hz}$ frequencies at $730 \mathrm{~V}$ by hexagonal needle under general anesthesia. The necrosis of the remaining breast resulted in a voluminous eschar that was easily removed few months after leaving the chest wall free from macroscopic disease turning in a non-surgical mastectomy.

Conclusions. This kind of breakthrough application of electrochemotherapy might be considered to avoid palliative mastectomy in very selected patients. New technologies may help clinicians to find agreement between patient' will and the burden of treatment and might contribute in selected cases to give options to patients not keen on having surgery.

\section{Background}

The incidence of multiple primary malignant neoplasms has increased during the last decades as screening program has been implemented and cancer therapies have become more effective.

Since first described by Billroth in 1889(1) and after reviewed by Warren and Gates in 1932 (2), specific guidelines have been drawn, however multiple neoplasms can translate in multiple drug resistance which drives clinicians to opt for different strategies(3).

Breast cancer is the most common cause of cancer amongst women(4). The risk of developing breast cancer increases with age. The gold standard for the treatment of early breast cancer is breast conserving surgery along with radiation therapy. The 15-year absolute risk of breast cancer death is $3.8 \%(95 \% \mathrm{Cl}$ $1 \cdot 6-6 \cdot 0,2 p=0 \cdot 00005$ ). The 10-year risk of any (loco regional or distant) first recurrence is $19.3 \%$ if radiation treatment follows surgery(5).

In 5 to $30 \%$ local recurrence appears with synchronous skin metastases(6). Skin metastases do not impact on overall survival, however they have a major impact on the quality of life of patients causing pain, ulceration, infection, and psychological discomfort. Systemic treatments often fail to provide adequate local control of the disease. The most of patient are not eligible for resection and frequently develop resistance to any other systemic therapy(6). 
Electrochemotherapy (ECT) has shown encouraging results amongst patients with skin metastases from breast and other types of solid cancers(7-9). ECT combines the administration of antineoplastic drugs such as cisplatin or bleomycin (6) with electroporation of tumor cells (6), intraoperatively. Electric pulses permeabilize the cell membrane and allow the drug to penetrate the cell and increase cytotoxicity. The codification of the procedure by European Standard Operating Procedure (ESOPE) guidelines in 2006(10) led to a broad diffusion of the procedure, mainly in European centers, and since then, the progressive clinical experience, together with the emerging technologies, have extended its application to new groups of patients(7).

ECT is the gold standard treatment of patients with cutaneous metastases $(6,7)$ who, due to the extent of their metastases, are not eligible for resection or radiotherapy has already been used, and systemic therapy is ineffective or contraindicated. Several clinical studies have demonstrated that ECT provides safe, effective, and non-invasive loco-regional treatment for chest wall breast cancer recurrence $(11,12)$.

Here we present an extended indication to electrochemotherapy for breast cancer recurrence after breast conserving surgery. Electroporation-induced necrosis replaced surgery and was delivered to treat breast cancer recurrence in multi-drug resistant patient who refused standard surgery. The effect was a massive necrosis of the remaining breast that resulted in a non-surgical mastectomy. This is one of a kind different application of electrochemotherapy and gives the chance to avoid palliative surgery in very selected patients.

\section{Case Presentation}

A 72 years old woman with a personal history of breast, lung and rectal cancer presented with an ulcerated breast local recurrence on her right breast. She had no familial history of breast and ovarian cancer and refused any genetic testing. In 1994, when she was 46 and premenopausal, she was diagnosed with hormone receptor positive breast cancer, no special type (NST). She had breast conserving surgery and axillary clearance along with twenty-five applications of conventional external beam radiation therapy. Anatomic stage by TNM was pT2NOMO. She was prescribed five years endocrine therapy with tamoxifen and ovarian suppression with luteinizing hormone-releasing hormone (LHRH) agonists.

In 2010 she was diagnosed with right non-small cell lung cancer (NSCLC) complicated by pleural effusion. Stage at diagnosis was T2N1M1a. She was treated with first line chemotherapy 4 cycles of cisplatinum and pemetrexed and obtained partial response. Ten months later, she had a local relapse. She was screened for epidermal growth factor receptor mutation and deletion of exon 19 (Ex19Del) was found. Thus she started with EGFR tyrosine kinase inhibitor (TKI) gefitinib.

Since August 2013, following mediastinal lymph node progression, the patient was administered platinum-based doublet chemotherapy. After further progression, patient was treated with third line with EGFR tyrosine kinase inhibitor (TKI) erlotinib -- and targeted radiotherapy on lung lesion. 
In 2015 she complained with abdominal pain. Rectal examination showed a rigid mass close to the dentate line. She underwent colonoscopy that revealed an ulcerated mass occupying half of the circumference of the colon -rectal lumen and rectal biopsy was performed. She was diagnosed with locally advanced rectal cancer and scheduled for neo-adjuvant chemo-radiotherapy. She had complete response and refused completion rectal surgery.

In 2016 she developed right in-breast lump complicated by mediastinal lymphadenopathy. Mammography and ultrasound scan were suspicious for breast local relapse, then confirmed by right breast core biopsy showing receptor positive HER2 amplified breast cancer (ER 85\%; PgR 40\%; Ki67 20\%; HER2 +++). She started weekly paclitaxel, antiHER2 monoclonal antibody trastuzumab every 21 days and oral vinorelbine. She developed peripheral neuropathy, asthenia and dyspeptic syndrome, thus suspended paclitaxel and kept on with trastuzumab. Disease was deemed stable after six months follow up.

In 2018, lung cancer progressed therefore patient underwent 45 Gy Cyber-knife System radiotherapy. The PET-CT scan showed good response on the lung.

In 2019 rectal cancer recurred and underwent robotic Miles' abdominoperineal resection surgery and left colostomy. At the same time breast recurrence progressed with skin ulceration, redness and infiltration of pectoralis muscle (Fig. 1).

The infiltrated area was eight centimeter large and extended all over the right hemi-thorax.

Multidisciplinary meeting (MDM) went through different surgical options. Modified radical mastectomy without tissue flap reconstruction was excluded as there was not enough skin to bridge the gap. Skin salvage mastectomy and reconstructive tissue flap was first offered to patient who refused that.

After a second MDM electrochemotherapy on the breast was proposed. Patient was explained about risks and benefit of the procedure and signed the informed consent. She was scheduled for electrochemotherapy on the right breast under general anesthesia. After intravenous injection of bleomycin $15 \mathrm{mg} / \mathrm{m}^{2}, 101$ pulses at $5000 \mathrm{~Hz}$ frequencies were delivered by hexagonal needle electrode $40 \mathrm{~mm}$ length, at 730V voltage with Cliniporator ${ }^{\text {TM }}$ (IGEA Srl, Carpi [MO], Italy). The treatment was administered following the ESOPE guidelines(10). The procedure lasted 30 minutes. At the end of the procedure greasy meshed gauzes dressing were applied. After treatment patient was brought in the postanesthetic recovery room and then to the ward. Patient was discharged the day after in good condition. The post-treatment follow up was scheduled on day 7th - 15th - 30th -60th - 90th (Fig. 2; Fig. 3). After ninety days the whole breast was replaced by necrotic tissue as shown in Fig. 4. Escharotomy to remove the necrotic tissue was performed under local anesthesia, leaving the chest wall free from macroscopic disease and the result was a mastectomy induced by electrochemotherapy. In order to evaluate the residual burden of disease after escharotomy we performed multiple punch biopsies on the living tissue underneath the eschar. Pathology report showed few residual of breast cancer cells surrounded by disease-free margins (Fig. 5). Advanced dressings were applied during follow up. After two months the wound closed (Fig. 6). Six months after the wound closed the patient was clinically free from disease on 
the chest wall. PET-CT scan showed non-evident disease from rectal cancer whereas lung metastases are stable. Patient is satisfied with the procedure and continues her routinely follow up with improved quality of life.

\section{Discussion And Conclusions}

It is not uncommon for people with severe cancer to refuse treatment, even when that decision might worsen their prognosis. However, new technologies may help clinicians to find agreement between patient' will and the burden of treatment. Here we have presented the case of a women treated for multiple primary cancers whose prognosis has been worsened by several local and distant relapses and resistant to different drugs. After more than twenty years of cancer fighting the patient had the right to refuse surgery consisting of mastectomy and flap reconstruction that she considered a major surgery to deal with. On the other hand her quality of life was significantly impaired by ulcerative and exudative breast cancer recurrence that required daily dressing and frequently antibiotics cycling.

It is well-established electrochemotherapy represent the gold standard treatment of patients with cutaneous metastases $(6,7)$ and it is now a breach of duty not to offer electrochemotherapy to those patients. Nevertheless, this was not the case. Skin ulceration, malodorous and swelling lump need to be treated with mastectomy in order to improve patient quality of life. However, since the patient refused surgery we considered electrochemotherapy as a possible option to replace debulking mastectomy. The electroporation-induced necrosis fulfilled our expectations by creating a voluminous eschar involving the whole breast, easily removed by escharotomy under local anesthesia. Electrochemotherapy is a fast procedure that can be easily delivered in about 30 minutes either under local and general anesthesia. The recovery is quick compared to major surgery such as breast and chest wall reconstruction. As far as we know, this is the first time electrochemotherapy has replaced debulking mastectomy after breast conserving surgery and should be considered as one of a kind breakthrough application of this technology in very selected cases, specifically amongst patients. Although someone can argue the healing process is quite long, the patient was greatly happy to have been spared from major surgery. She could change her dressing easily at home and felt very confortable with the procedure. This case report might represent a step forward to give options to very selected patients not keen on having breast surgery after recurrence.

\section{List Of Abbreviations}

CT: Computed Tomography; ECT: Electrochemotherapy; EGFR: Epidermal ER: Estrogen Receptor; Growing Factor Receptor; ESOPE: European Standard Operating Procedure; HER: Human Epidermal Growth Factor Receptor; Hz: Hertz Gy: Grays; LHRH: Luteinizing hormone-releasing hormone; NSCLC: non-small cell lung cancer; PET: Positron Emission Tomography; PgR: Progesterone Receptor; TKI: Tyrosine Kinase Inhibitor; V: Volt

\section{Declarations}




\section{Ethics approval and consent to participate}

Manuscript received approval from Internal Ethic Committee

\section{Consent for publication}

Written informed consent was obtained from the patient for publication of this Case report and any accompanying images. A copy of the written consent is available for review by the Editor of this journal.

\section{Availability of data and materials}

The datasets used and/or analyzed during the current study are available from the corresponding author on reasonable request.

\section{Funding}

Not applicable

\section{Authors' contributions}

EE was a major contributor in writing the manuscript

CS analyzed and interpreted the patient data

UP analyzed and interpreted the patient data

$\mathrm{RC}$ analyzed patient data and contributed in writing the manuscript

RdG conceived the idea of the manuscript and interpreted patient data

All authors read and approved the final manuscript

\section{Acknowledgements}

Authors acknowledge anyone who contributed towards the article who does not meet the criteria for authorship.

\section{References}

1. Billroth T. Die allgemeine chirurgische pathologie and therapie.1889. $14 \mathrm{p}$.

2. S. W. Multiple primary malignant tumors: a survey of the literature and a statistical study. American Journal of Cancer. 1932.

3. Markakis C, Marinis A, Dikeakos P, Grivas P, Voultsos M, Liarmakopoulos E, et al. Multiple synchronous primary neoplasms of the breast, colon and rectum after surgery for endometrial cancer: A case report. Int J Surg Case Rep. 2013;4(5):493-5. 
4. Cuzick J, Stewart H, Rutqvist L, Houghton J, Edwards R, Redmond C, et al. Cause-specific mortality in long-term survivors of breast cancer who participated in trials of radiotherapy. J Clin Oncol. 1994;12(3):447-53.

5. Darby S, McGale P, Correa C, Taylor C, Arriagada R, Clarke M, et al. Effect of radiotherapy after breastconserving surgery on 10-year recurrence and 15-year breast cancer death: meta-analysis of individual patient data for 10,801 women in 17 randomised trials. Lancet. 2011;378(9804):1707-16.

6. Wichtowski M, Murawa D, Kulcenty K, Zaleska K. Electrochemotherapy in Breast Cancer - Discussion of the Method and Literature Review. Breast Care (Basel). 2017;12(6):409-14.

7. Campana LG, Edhemovic I, Soden D, Perrone AM, Scarpa M, Campanacci L, et al. Electrochemotherapy - Emerging applications technical advances, new indications, combined approaches, and multi-institutional collaboration. Eur J Surg Oncol. 2019;45(2):92-102.

8. Kunte C, Letulé V, Gehl J, Dahlstroem K, Curatolo P, Rotunno R, et al. Electrochemotherapy in the treatment of metastatic malignant melanoma: a prospective cohort study by InspECT. Br J Dermatol. 2017;176(6):1475-85.

9. Bertino G, Sersa G, De Terlizzi F, Occhini A, Plaschke CC, Groselj A, et al. European Research on Electrochemotherapy in Head and Neck Cancer (EURECA) project: Results of the treatment of skin cancer. Eur J Cancer. 2016;63:41-52.

10. al GJe. Results of the ESOPE (European Standard Operating Procedures on Electrochemotherapy) study: Efficient, highly tolerable and simple palliative treatment of cutaneous and subcutaneous metastases from cancers of any histology. Journal of Clinical Oncology. 2006;24(18).

11. Matthiessen LW, Keshtgar M, Curatolo P, Kunte C, Grischke EM, Odili J, et al. Electrochemotherapy for Breast Cancer-Results From the INSPECT Database. Clin Breast Cancer. 2018;18(5):e909-e17.

12. Esmaeili N, Friebe M. Electrochemotherapy. A Review of Current Status, Alternative IGP Approaches, and Future Perspectives. J Healthc Eng. 2019;2019:2784516.

\section{Figures}




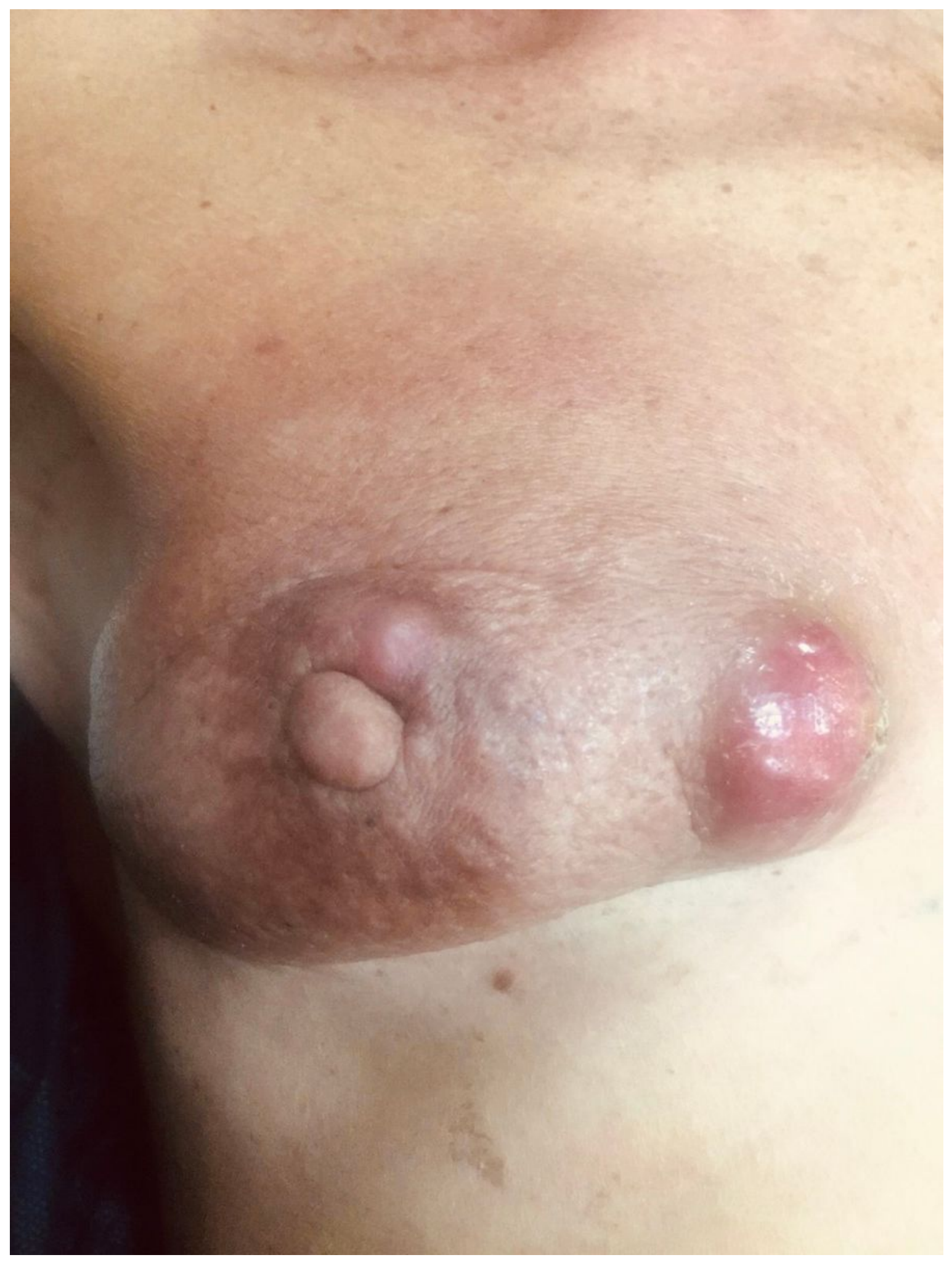

\section{Figure 1}

Preoperative image. Right breast cancer recurrence with skin ulceration, swelling and redness. 


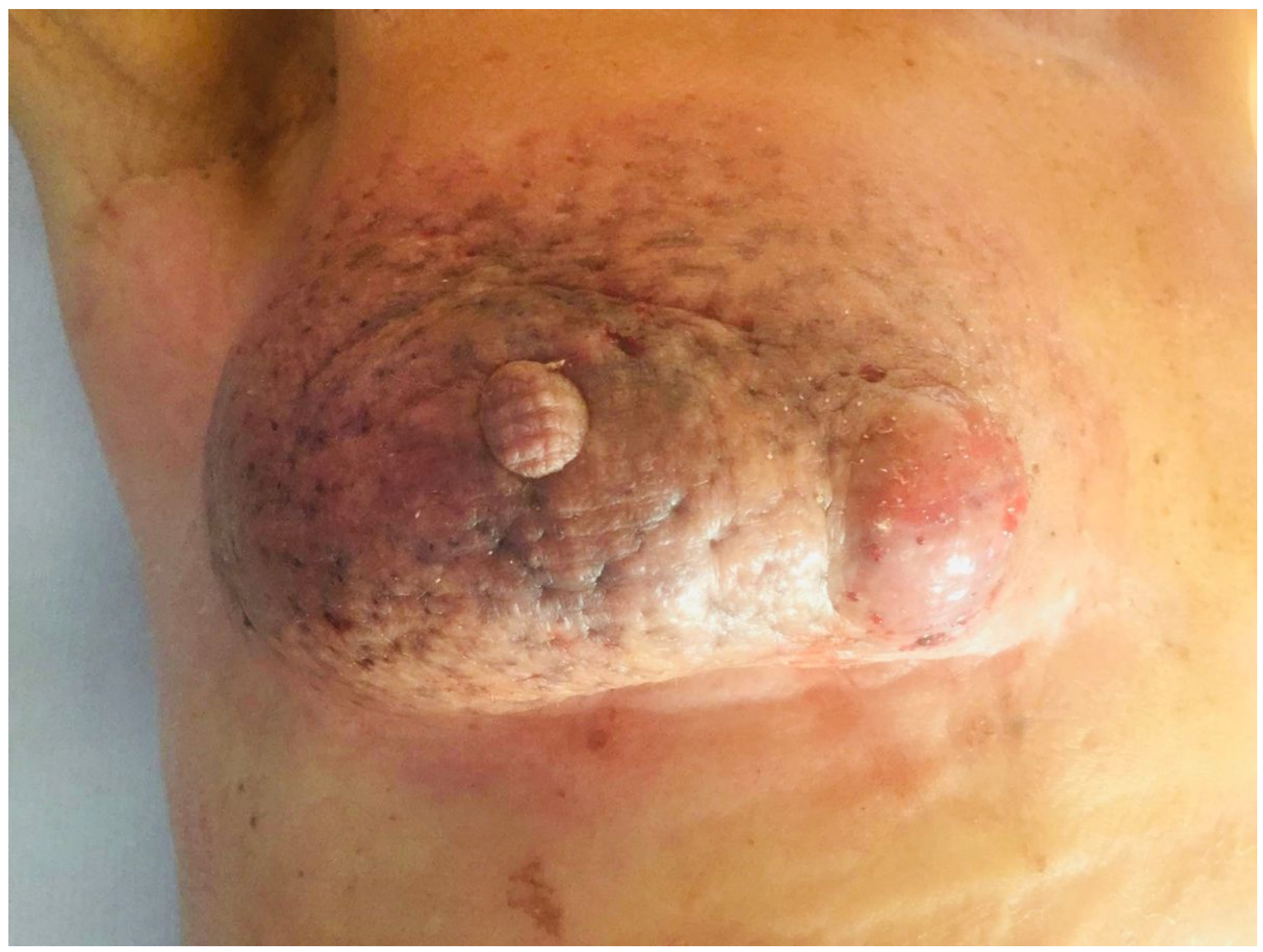

Figure 2

Post-operative image. Right breast thirty days after electrochemotherapy 


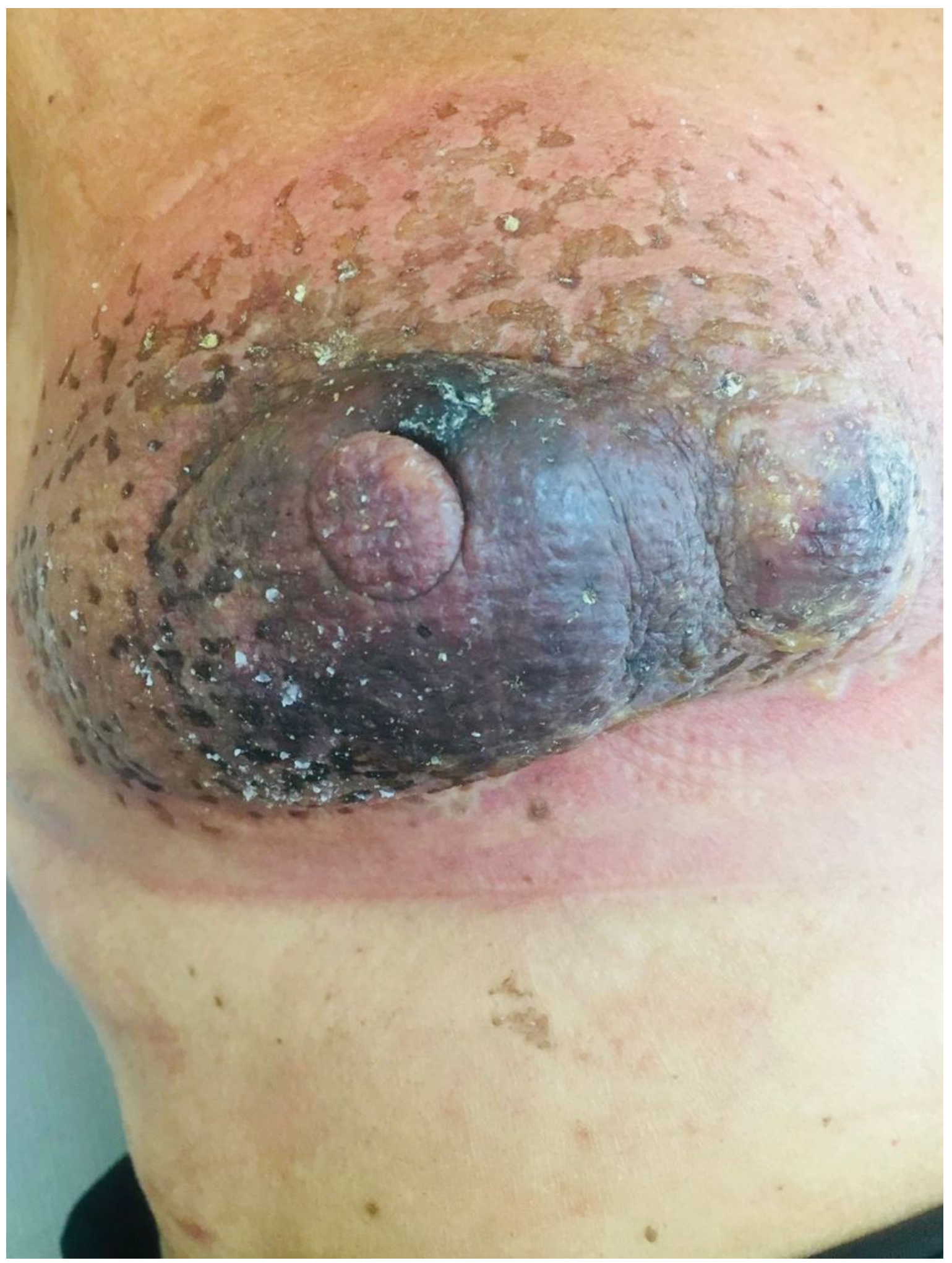

Figure 3

Post-operative image. Right breast sixty days after electrochemotherapy 


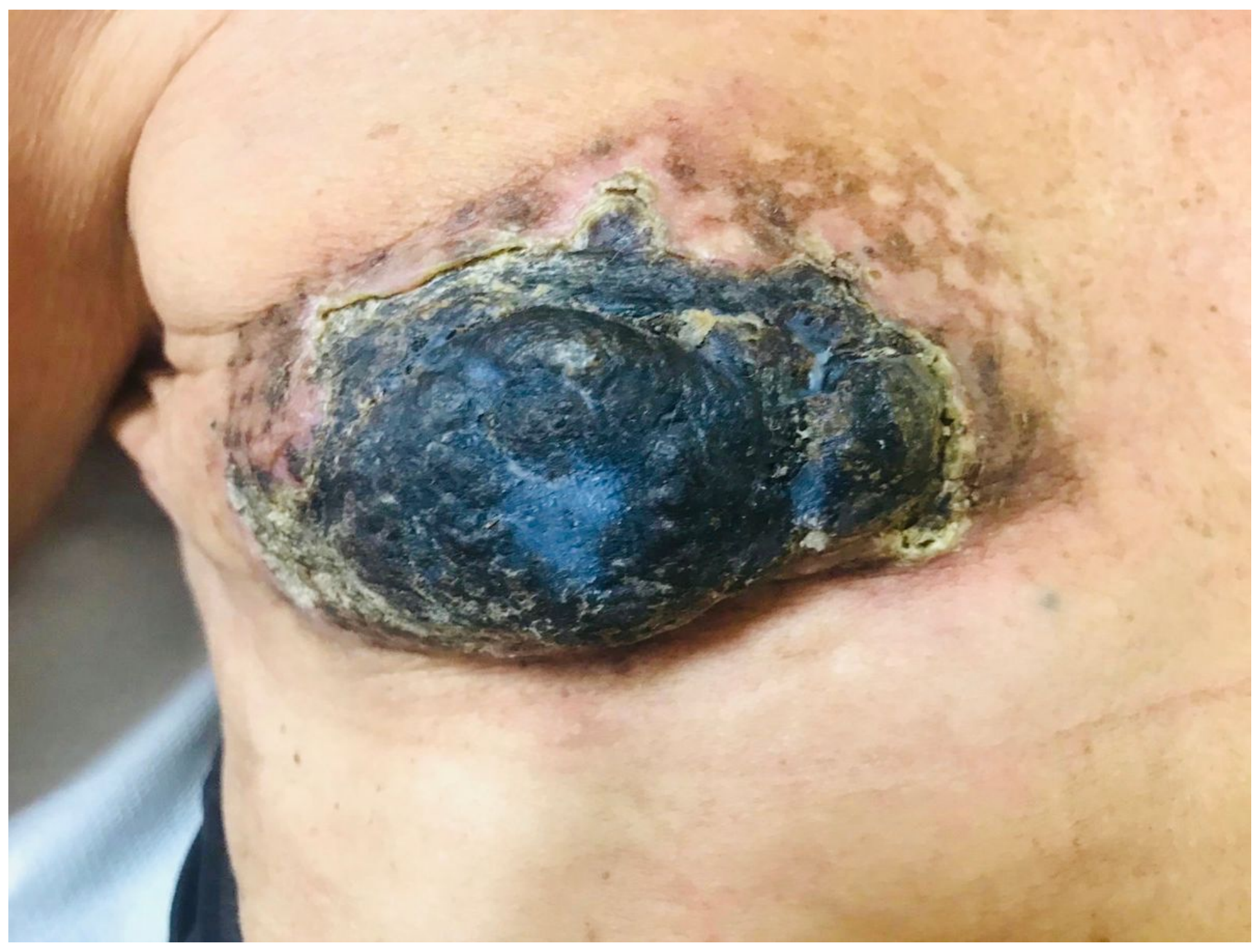

Figure 4

Post-operative image. Right breast ninety days after electrochemotherapy 


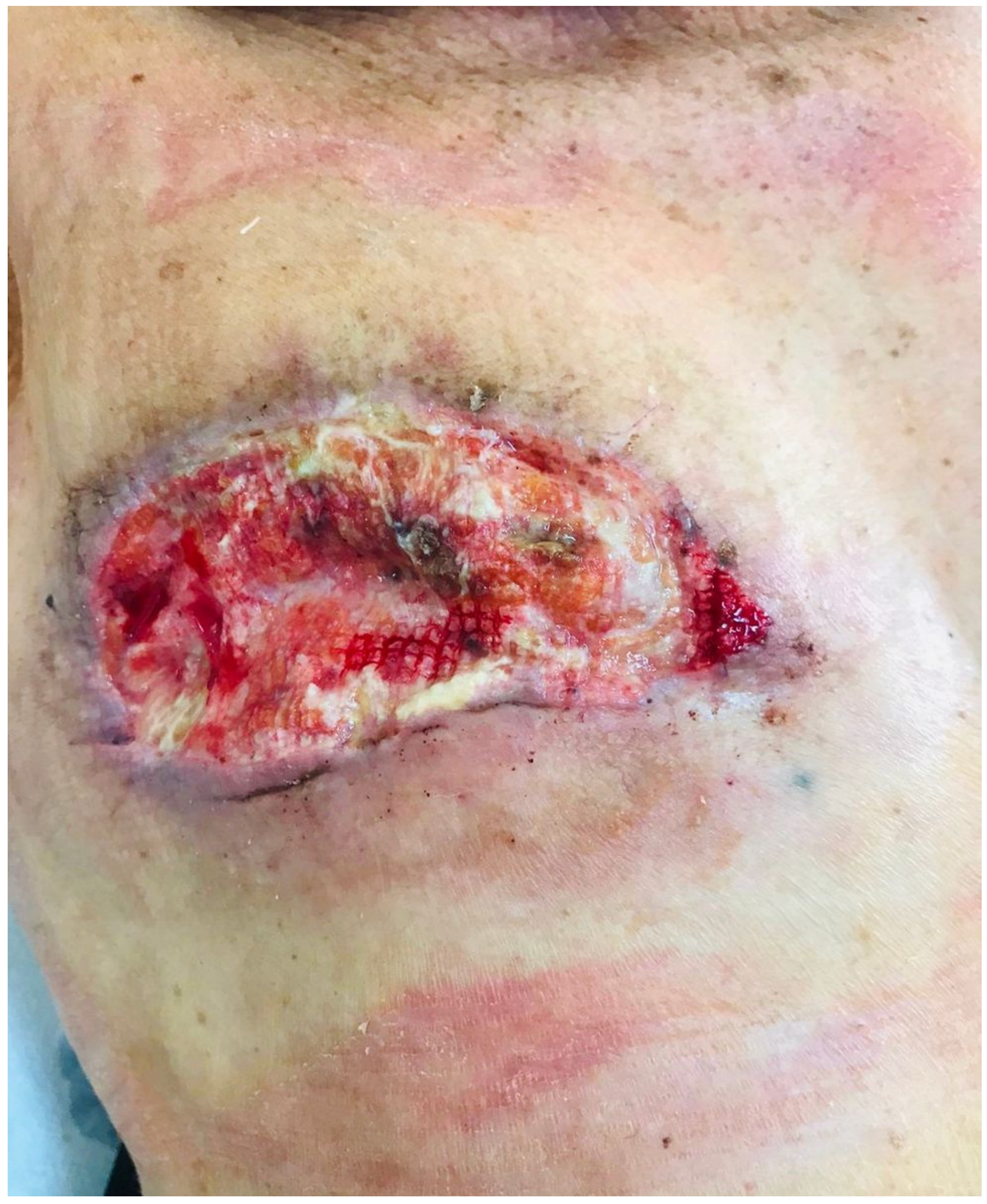

Figure 5

Post-operative image. Right side chest wall one day after escharotomy 


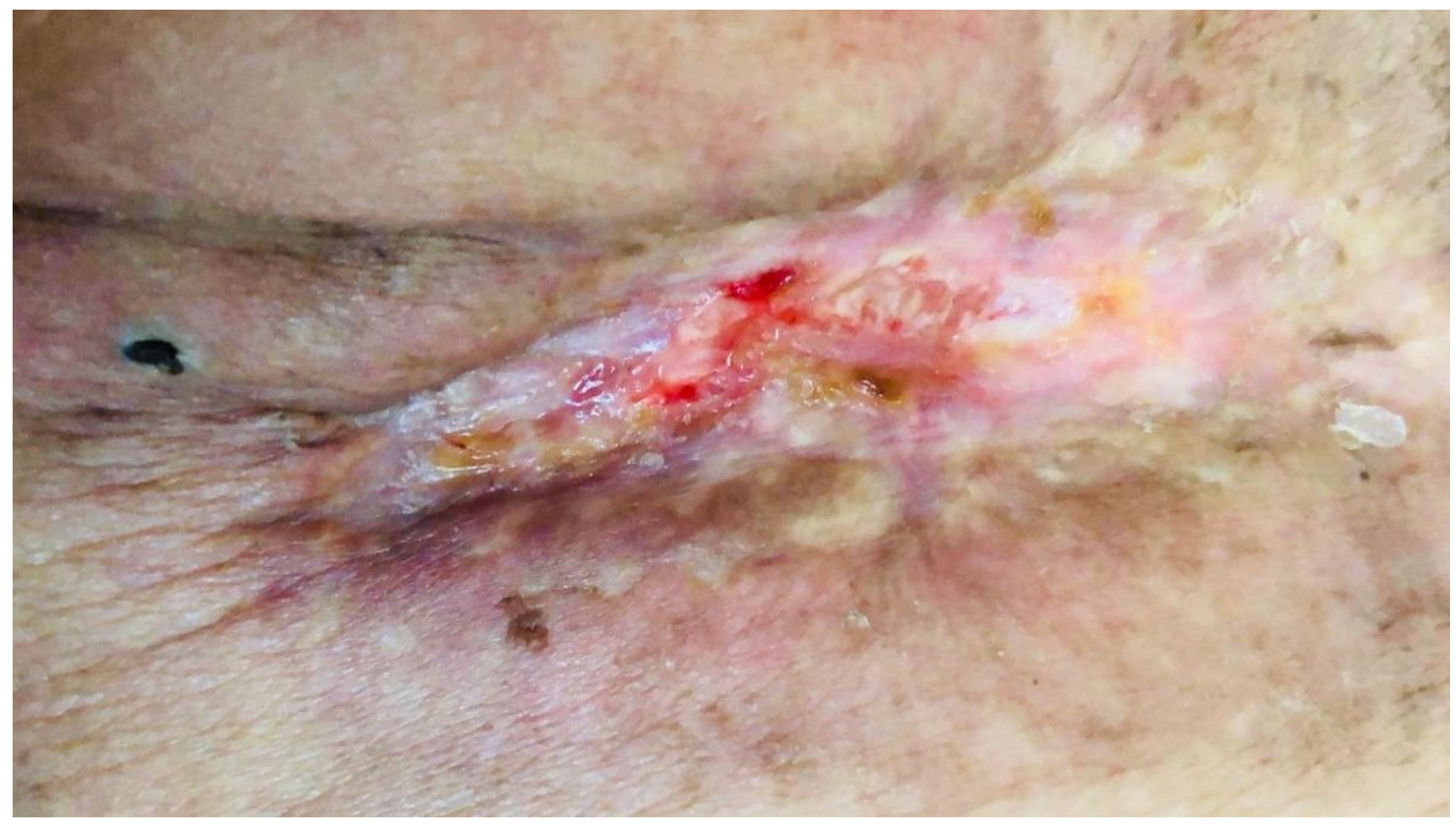

Figure 6

Wound closure sixty days after escharothomy

\section{Supplementary Files}

This is a list of supplementary files associated with this preprint. Click to download.

- CAREchecklistEnglish2013.pdf 